International Mathematical Forum, 1, 2006, no. 24, 1175 - 1180

\title{
ON MULTIPLICATION MODULES
}

\author{
S. Ebrahimi Atani and S. Khojasteh G. Ghaleh \\ Department of Mathematics, University of Guilan \\ P. O. Box 1914, Rasht, Iran
}

\begin{abstract}
Let $R$ be a commutative ring with non-zero identity. This paper is devoted to study some of properties of multiplication modules. A number results concerning multiplication modules are given.
\end{abstract}

Mathematics Subject Classification: 13C05, 13C13, 13A15

Keywords: Multiplicatin modules, Strongly pure submodules

\section{Introduction}

Throughout this paper all rings will be commutative with non-zero identity and all modules will be unitary. Multiplication modules and ideals have been investigated in El-Bast and Smith (1988), P. F. Smith (1988) and others. The aim of this paper is to study multiplication modules over commutative rings. Various properties of multiplication modules are considered (see sec. 2).

Now we define the concepts that we will use. If $R$ is a ring and $N$ is a submodule of an $R$-module $M$, the ideal $\{r \in R: r M \subseteq N\}$ will be denoted by $(N: M)$. Then $(0: M)$ is the annihilator of $M$. An $R$-module $M$ is called a multiplication module if for each submodule $N$ of $M, N=I M$ for some ideal $I$ of $R$. In this case we can take $I=(N: M)$.

Let $N$ be an $R$-submodule of $M$. Then $N$ is pure in $M$ if any finite system of equations over $N$ which is solvable in $M$ is also solvable in $N$. So if $N$ is pure in $M$, then $I N=N \cap I M$ for each ideal $I$ of $R$. A module $M$ is pureinjective if and only if any (infinite) system of equations (allowing infinitely many indeterminates) in $M$ which is finitely solvable in $M$ is solvable in $M$. An $R$-module $M$ is regular if all its submodules are pure. An $R$-module is absolutely pure if it is pure in every module that contains it as a submodule. An important property of regular rings is that every module is absolutely pure (see [7]. 


\section{Multiplication modules}

Let $p$ be any prime and let $M$ denote the $Z$-module $E(Z / p Z)$, the injective hull of the $Z$-module $Z / p Z$. Then $M$ is not multiplication, but it is divisible. Moreover, the $Z$-module $Z / p^{n} Z, n \geq 1$, is multiplication, but it is not divisible. Now we will prove the following results:

Theorem 2.1. Let $M$ be an divisible module over an integral domain $R$. Then $M$ is multiplication if and only if $M$ is simple (so cyclic).

Proof. Clearly, if $M$ is simple then $M$ is multiplication. Conversely, assume that $M$ is multiplication and let $N$ be a non-zero submodule of $M$. Then there exists an ideal $I$ of $R$ such that $N=I M=M$, as required.

Since by [2, Corollary 4], over a commutative ring $R$ a simple module is pure-injective, we have the following corollary by Theorem 2.1:

Corollary 2.2. Over an integral domain $R$ a divisible multiplication module is pure-injective.

An $R$-module $M$ is secondary if $0 \neq M$ and, for each $r \in R$, the $R$ endomorphism of $M$ produced by multiplication by $r$ is either surjective or nilpotent. We recall that by [5. Theorem 2.2], over a commutative $\operatorname{ring} R$, every secondary multiplication $R$-module is finitely generated. Also, in general, every submodule of a finitely generated $R$-module is not finitely generated, but we have the following result:

Theorem 2.3. Every pure submodule of a secondary multiplication $R$ module is finitely generated.

Proof. Assume that that $M$ is a secondary multiplicatin $R$-module and let $N$ be a pure submodule of $M$, so by [6, Lemma 2.1], $N$ is a secondary submodule of $M$. Now we show that $N$ is multiplication. Let $K$ be a submodule of $N$. Then $K=I M$ for some ideal $I$ of $R$, so $I N=N \cap I M=N \cap K=K$ since $N$ is pure in $M$; hence $N$ is multiplication. Now the assertion follows from [5, Theorem 2.2].

Theorem 2.4. Let $R$ be a commutative ring and $M$ a finitely generated faithful multiplication $R$-module. Then $R$ is a regular ring if and only if $M$ is regular.

Proof. If $R$ is regular, then every module over $R$ is absolutely pure by [7, Theorem 16.A]. Conversely, suppose that $I$ is an ideal of $R$. It is enough to show that $I^{2}=I$. As $M$ is regular, $N=I M$ is a pure submodule of $M$, so 
$I N=I^{2} M=N \cap I M=I M$. It follows from [8, p. 231 Corollary] that $I^{2}=I$, as required.

Let $P$ be a maximal ideal of a ring $R$. An $R$-module $M$ is called $P$-torsion provided for each $m \in M$ there exists $p \in P$ such that $(1-p) m=0$. Also, an $R$-module $M$ is called prime if $\left(0:_{R} N\right)=\left(0:_{R} M\right)$ for every non-zero submodule $N$ of $M$.

Let $M$ denote the $Z$-module $Z / 2 Z \oplus Z / 3 Z$. Then $M$ is multiplication, but it is not a prime $Z$-module and also $Q$, the quotient field of $Z$, is prime which is not multiplication.

Proposition 2.5. Let $R$ be a commutative ring, $M$ a faithful prime multiplication $R$-module and $P$ a maximal ideal of $R$. If $M$ is $P$-torsion, then $M=0$.

Proof. Suppose that $M$ is $P$-torsion, so $M=P M$ by [9, Lemma 6]. We show that $M=0$. Otherewise, there exist $0 \neq m \in M$ and $p \in P$ such that $(1-p) m=0$, so $1-p \in\left(0:_{R} m\right)=\left(0:_{R} M\right)=0$ which is a contradiction. Thus $M=0$.

A submodule $N$ of a module $M$ is called strongly pure, $s$-pure for short, if for every finite tuple $x_{1}, x_{2}, \ldots, x_{n}$ of elements in $N$ there is a map $f: M \rightarrow N$ such that $f\left(x_{i}\right)=x_{i}, 1 \geq i \geq n$ (see [9]). It is easy to see that $s$-pure submodules are pure submodules, but the converse is not true. However, by [9, p. 339], we have the following lemma:

Lemma 2.6. Let $R$ be a commutative ring, $M$ a free $R$-module, and $N$ an $R$-submodule of $M$. Then $N$ is a s-pure submodule of $M$ if and only if it is a pure submodule of $M$.

Lemma 2.7. Let $R$ be a commutative ring, $M$ a free multiplication $R$-module, and $N$ a non-zero s-pure $R$-submodule of $M$. Then $I(N: M)=(N: M) \cap I$ for every ideal $I$ of $R$.

Proof. Assume that $I$ is an ideal of $R$ and let $\left\{x_{i}: i \in J\right\}$ be a basis of $M$, so $I N=N \cap I M$ since $N$ is pure by Lemma 2.6. As $I(N: M) \subseteq(N: M) \cap I$ is trivial, we will prove the reverse inclusion. Let $a \in(N: M) \cap I$. Then $a x_{i} \in I M \cap(N: M) M$, so $a x_{i} \in I(N: M) M=(N: M) M \cap I M$ since $M$ is multiplication. It follows that there exisit $c \in I$ and $d \in(N: M)$ such that $a x_{i}=c d x_{i}$; hence $a=c d \in I(N: M)$, as required.

Proposition 2.8. Let $R$ be a commutative ring, $M$ a free multiplication $R$ module, and $N$ a non-zero s-pure $R$-submodule of $M$. Then the ideal $(N: M)$ is idempotent. 
Proof. By Lemma 2.7, $(N: M)^{2}=(N: M) \cap(N: M)=(N: M)$.

Let $M$ be an $R$-module and $N$ be a submodule of $M$ such that $N=I M$ for some ideal $I$ of $R$. Then we say that $I$ is a presentation ideal of $N$. Note that it is posible that for a submodule $N$, no such presentation ideal exist. For example, If $M$ is a vector space over an arbitrary field $F$ with $\operatorname{dim}_{F}(M)=k \geq$ 2, then $M$ has finite length (so $M$ is notherian and artinian), but any proper subspace $N \neq 0$ of $M$ does not have any presentation. Moreover, $M$ is not multiplication. Clearly, every submodule of $M$ has a presentation ideal if and only if $M$ is a multiplication module.

Let $N$ and $K$ be submodules of a multiplication $R$-module $M$ with $N=$ $I_{1} M$ and $K=I_{2} M$ for some ideals $I_{1}$ and $I_{2}$ of $R$. The product $N$ and $K$ denoted by $N K$ is defined by $N K=I_{1} I_{2} M$. Then by [1, theorem 3.4], the product of $N$ and $K$ is independent of presentation of $N$ and $K$. Clearly, $N K$ is a submodule of $M$ and $N K \subseteq N \cap K$. Now we have the following result:

Theorem 2.9. Let $M$ be a free multiplicatin module over a commutative ring $R$. Then every non-zero s-pure submodule of $M$ is idempotent.

Proof. Let $N$ be a non-zero $s$-pure submodule of $M$. Then by Proposition 2.8, we have $N^{2}=(N: M)^{2} M=(N: M) M=N$.

A proper submodule $N$ of a module $M$ over a commutative ring $R$ is said to be weakly primary submodule (resp. primary submodule) if whenever $0 \neq r m \in N$ (resp. $r m \in N$ ), for some $r \in R, m \in M$, then $m \in N$ or $r^{n} M \subseteq N$ for some $n$. So a primary submodule is weakly prime. However, since 0 is always weakly prime (by definition), a weakly prime submodule need not be prime, but we have the following result:

Theorem 2.10. Let $R$ be a commutative ring, $M$ a free multiplication $R$ module, and $N$ a non-zero s-pure $R$-submodule of $M$. Then $N$ is primary submodule of $M$ if and only if it is weakly primary submodule of $M$.

Proof. It is enough to show that if $N$ is weakly primary then $N$ is primary. Assume that $N \neq 0$ is a weakly primary submodule of $M$ that is not primary. Then by [4, Proposition 2.14] and Proposition 2.8, we have $N=(N: M) M=$ $(N: M)^{2} M=(N: M) N=0$ which is a contradiction. Thus $N$ is primary.

Proposition 2.11. Let $R$ be a commutative Noetherian ring with Jacobson radical $J(R), M$ a free multiplication $R$-module, and $N$ a non-zero s-pure $R$ submodule of $M$. Then There is a maximal ideal $P$ of $R$ such that $(N: M) \nsubseteq$ $P$.

Proof. Otherwise, $(N: M) \subseteq J(R)$, so $(N: M)=\bigcap_{i=1}^{\infty}(N: M)^{i}=0$ by Proposition 2.8; hence $N=(N: M) M=0$ which is a contradiction. 
Proposition 2.12. Let $R$ be a commutative ring with Jacobson radical $J(R)$, $M$ a finitely generated $R$-module, and $N$ a finitely generated multiplication $R$ submodule of $M$. If $N$ is a s-pure submodule of $M$ under the map $f: M \rightarrow N$ with $J(R) M=\operatorname{Ker}(f)$, then $M$ is a multiplication module.

Proof. Let $N=R n_{1}+R n_{2}+\ldots+R n_{k}$. Then by $s$-purity of $N$, for each $i=1, \ldots, k$, we have $f\left(n_{i}\right)=n_{i}$, so $M / J(R) M=M / \operatorname{Ker}(f) \cong N$ is a multiplicatin $R$-module. Now the assertion follows from [3, Corollary 1.5].

Compare the next result with [3, Theorem 3.5].

Theorem 2.13. Let $R$ be a commutative ring with Jacobson radical $J(R), M$ a non-zero finitely generated $R$-module, and $N$ a finitely generated multiplication s-pure submodule of $M$ under the map $f: M \rightarrow N$ with $K=\operatorname{Ker}(f) \subseteq$ $J(R) M$. Then an $R$-submodule $L$ of $M$ is a maximal submodule if and only if there exists a maximal ideal $P$ of $R$ such that $L=P M \neq M$.

Proof. Let $L$ be a maximal submodule of $M$. We show that $K=\operatorname{Ker}(\mathrm{f}) \subseteq$ $L$. Otherwise, there is an element $x \in K$ such that $x \notin L$, so $M=L+R x \subseteq$ $L+J(R) M$; hence $M=L+J(R) M$. It follows from Nakayama's lemma that $M=L$ which is a contradiction. Thus $K \subseteq L$. We divided the proof into the following two cases:

Case $1 K=L$.

Since $K \subseteq J(R) M$, we obtain $K=J(R) M$ and $M \neq J(R) M$, so by Proposition 2.12, $M$ is a multiplication module; hence by [3, Theorem 2.5], there is a maximal ideal $P$ of $R$ such that $L=P M \neq M$.

Case $2 K \neq L$.

Since $M / K \cong N$ is a non-zero multiplication $R$-module and $L / K$ is a maximal submodule of $M / K$, by [3, Theorem 2.5], there exists a maximal ideal $Q$ of $R$ such that $L / K=Q(M / K)=(Q M+K) / K=Q M / K \neq M / K$; hence $L=Q M \neq M$.

Conversely, assume that $P$ is a maximal ideal of $R$ such that $P M \neq M$. Then $P(M / K)=(P M+K) / K=P M / K \neq M / K$, so $P M / K$ is a maximal submodule of the multiplication $R$-module $M / K$; hence $P M$ is a maximal submodule of $M$, as required.

\section{References}

[1] R. Ameri, On the prime submodules of multiplication modules, International Journal of Mathematics and Mathematical Sciences, 27 (2003), 1715-1724.

[2] T. J. Cheatham and J. R. Smith, regular and semisimple modules, Pacific J. of Math., 2 (1976), 315-323. 
[3] Z. A. El-Bast and P. F. Smith, Multiplication modules, Comm. in Algebra, 16 (1988), 755-779.

[4] S. Ebrahimi Atani, The product of multiplication submodules, Honam Math. J., 27 (2005), 1-8.

[5] S. Ebrahimi Atani, Multiplication modules and related results, Archivum Mathematicum, 40 (2004), 407-414.

[6] S. Ebrahimi Atani, On secondary modules over Dedekind domains, Southeast Asian Bull. of Math., 25 (2001), 1-6.

[7] M. Prest, Model Theory and Modules, London Mathematical Society, Lecture Note Series, Cambridge: Cambridge Univercity Press, 1988.

[8] P. F. Smith, Some remarks on multiplication module, Arch. Math., 1988 50, 223-235.

[9] W. Zimmermann, On locally pure-injective modules, J. of Pure and Applied Algebra, 166 (2002), 337-357.

Received: October 23, 2005 